\title{
Universe: the dynamics of the minimum and maximum expansion states
}

Sócrates Petrakis ( $\square$ sgeorgespp@gmail.com )

S.E.E.-M.G. https://orcid.org/0000-0001-7549-1009

Physical Sciences - Article

Keywords: Universe, Energy, Spin, Gravity, Entropy

Posted Date: July 13th, 2021

DOl: https://doi.org/10.21203/rs.3.rs-708548/v1

License: (c) (i) This work is licensed under a Creative Commons Attribution 4.0 International License. Read Full License 
Universe: the dynamics of the minimum and maximum expansion states

\author{
Sócrates Georges Petrakis \\ SEEMG, Belo Horizonte - MG, Brazil \\ Physicist Licensed (UFMG) \\ ORCID ID: 0000 - 0001 - 7549 - 1009 \\ E-mail: sgeorgespp@gmail.com
}

\begin{abstract}
Two hypotheses stand out in describing the evolution of the Universe. The predominant one predicts that the current expansion began at a certain instant and will not preserve any variation of energy that performs work; apparent flat Universe $(\Omega=1)$ is advocated by relativistic calculations and observational data, with an end or thermal death at its maximum expansion (3D space). The other hypothesis considers that the Universe is always cyclical (alternating phases of expansion and contraction). This proposal aims to demonstrate that both hypotheses can be correct by not being distinct, but complementary. Supported by the immutability of physical laws, analyses of concepts such as space, mass, energy, gravity, spin, and entropy define an exclusive presence of 1D space in the minimum and maximum expansion states of the Universe. With our 3D space Universe created and existing between these extreme states, a complete universal evolution is outlined. More objectively, all the exposed dynamics complements the usual relativity. The concept of complete rest energy (1D space) was able to be applied, demonstrating that the evolution of the entire Universe is spatially dynamic in a perpetual time dimension, always recreating our Universe.
\end{abstract}

Keywords: Universe, Energy, Spin, Gravity, Entropy. 


\section{Introduction}

The state of the Universe depends on energy. If the Universe is in an extremely contracted state, with the scarcity of empty space inside, no environment that promotes life would exist. In contrast, if the Universe is in a well-expanded state, energies would generate agglutinations for planetary formations or living beings; hence, the study of universal evolution [1] is important.

In Cosmology, the evolution of the Universe [2] is studied via astronomical observations, particle collision experiments, computational analysis, and relativistic calculations. However, its complete understanding is hindered by factors, such as the unknown nature of some of its constituents and real size.

Our solar system is billions of kilometers long and forms our galaxy together with billions of similar systems. The observable Universe alone has an incredibly large number of stars and galaxies.

Nonetheless, the most accepted theory about our Universe evolution posits a certain initial state and an accelerated expansion (space). However, the structure generating this expansive beginning is still under debate.

Investigations of the current expansion have shown that, in the beginning, everything occupied a minimum volume. Therefore, many studies associate this initial state with the extreme densities present in black holes [3]. Even these structures do not have all the mass and energy, or the constituents of the Universe and their composition are not well defined. Most studies have indicated the existence of an internal component, called singularity, but others have excluded this possibility through relativistic calculations $[4,5]$.

The instants of extreme universal contraction are usually related to presence of a great energy concentration.

Moreover, the existence of an expansive universe, the quantity of predicted massive current, and entropy (S) are commonly associated with a future thermal death or total dissipation.

Given these current associations, this study highlights concepts such as energy [6], dimension, and Universe, to present solutions in a coherent development. 


\section{Development}

The entire Universe is always the holder of any existence: mass and energy distributed in space (in transformation $\leftrightarrow$ Time); since there is nothing external for there to be exchange, the quantity of components must be conserved. Thus, there is certain variation in space with the flow of time.

The possible variation of Time relativity is always the same: limited by speed $(v=0$ and $v=c)$.

Limited variation of Time relativity $\leftrightarrow$ limited mass and energy concentration $\leftrightarrow$ limited variation of possible space $\leftrightarrow$ Limited contraction or expansion $\leftrightarrow$ Finite contraction and expansion energies $\leftrightarrow$ limited entire Universe.

Limited entire Universe: for the beginning or the end of any relative motion (expansion), it is necessary to be at rest.

Before the expansive beginning, the components of the entire universe had to be at rest.

For the maximum expansion, the components of the entire Universe will be at rest.

For the presence of extreme and static states, conserving the dynamic energy, the presence of maximum potential energy (the space vague between the masses) is necessary, i.e., the presence of a maximum distance (occupied or not) or 1D space.

The possible variation of space is always the same: limited by 1D space.

The entropy depends on the distribution of components in space and on the degree of freedom present, that is, on the type of space that exists: 3D, 2D or 1D; the degree of freedom is limited in $1 \mathrm{D}$ space.

Maximum contraction: 1D space has a maximum occupation; relative motion is zero; spin is zero.

Maximum expansion: 1D space has a minimum occupation; the space (between the two minimum 1D particles) is vague; spin is zero. 
There are studies that propose the existence of $1 \mathrm{D}$ and $2 \mathrm{D}$ space $[7,8]$.

Relativity (3D + 1D) and quantum mechanics analyze the beginning of the expansion only of our Universe (3D space); the initial instant of expansion of the entire universe happened earlier, in 1D space.

Energy (entire Universe) is neither created nor lost; energy is exchanged or transformed.

Since our Universe does not conserve its total energy (relativity), it is necessary the existence of internal matter (lower dimension) in 3D space, making the Universe as a whole, conserve its energy.

Mass formed by internal spaces (2D) is the existence in which one of the dimensions is minimum or no space for variation. Mass formed by internal spaces (1D) is the existence in which two of the dimensions are minimum, with no spaces for variation.

In the analysis of extreme static states (relative motion is zero), it is possible to apply the concept of rest energy.

When the internal energetic interactions of any amount of rest mass $\left(\mathrm{m}_{0}\right)$ are undone, all value of gravitational potential energy $(\mathrm{Eg})$, of energetic interactions: quantum, atomic and electromagnetic (Eq), and of internal kinetic energy (Ek) [9] are equivalent to the total rest energy $\left(E T=E_{0}=m_{0} \times c^{2}\right)[10]$.

The total invariant mass (at the resting instant of the universe: minimum or maximum expansion) is the analog of the resting mass.

Thus, ET (1D) is expressed as follows:

$$
E T=E_{0}=E g+E q+E k=m_{0} \times c^{2}
$$


For Universal internal resting state (minimum and maximum (1D) expansion), the following points are considered:

- $\mathrm{ETmaxC}=\mathrm{EgC}+\mathrm{EqC}+\mathrm{EkC}$, where ETmaxC, EgC, EqC and EkC are the maximum contraction total energy, gravitational potential, Eq, and Kinetic energies, respectively, in this extreme contracted state.

- $\mathrm{ETmax} E=\mathrm{EgE}+\mathrm{EqE}+\mathrm{EkE}$, where ETmaxE, EgE, EqE and EkE are the maximum expansion total energy, gravitational potential, Eq, and Kinetic energies, respectively, in this extreme expansive state.

- $E k C=0$ (for the state at rest, relative motion is zero): the maximum contraction.

- $\mathrm{EkE}=0$ (for the state at rest, relative motion is zero): the maximum expansion).

- $E q C=0(E k C=0$. Only static state (Quantum energy $(Q e)=$ Planck's constant $(h) \times$ Frequency $(F)=0(F=0 ; E k c=0)$. Without atomic presence, and without electromagnetic interactions).

- $\mathrm{EqE}=0(\mathrm{EkE}=0$. Only static state $(\mathrm{Qe}=\mathrm{h} \times \mathrm{F}=0 ; \mathrm{F}=0)$, without atomic presence, and without electromagnetic interactions).

- $\mathrm{ETmax}=\mathrm{ETmaxC}=\mathrm{EgE}$ (maximum value) $+\mathrm{EqE}(=0)+\mathrm{EkE}(=0)=$ $\mathrm{EgC}$ (maximum value) $+\mathrm{EqC}(=0)+\operatorname{EkC}(=0)$

- For maximum EgE, the maximum distance (the empty space) between the two minimum massive particles (one- dimensional) must be present.

- For $\mathrm{EgC}$, the maximum value must be present, even with the scarce empty spaces.

- For $\mathrm{EgC}$ to have the maximum value, the masses must be distributed in 1D length (maximum distance between the centers of mass). 
If for $\mathrm{EgC}$ the masses are distributed in $1 \mathrm{D}$ length $(\mathrm{d}=2 \mathrm{r})$, and for $\mathrm{EgE}$ the maximum distance (the empty space) between the two minimum massive particles is 1D, the gravitational field is uniform, and the reference point is the center of mass. Thus, for these extreme states, the use of the integral: $U(x)=\int \mathbf{F}(x) \cdot \mathrm{d} x=\mathrm{Eg}=(-\mathrm{G} \cdot \mathrm{m} . \mathrm{m} / \mathrm{r})$ makes the value of potential energy negative; with the value of Ek tending to zero or equal to zero (at minimum and maximum expansion), Eg has a maximum value tending to zero.

For uniform gravitational field (in these extreme states), total energies are positive $(E g=m . g . h ; g=$ acceleration of gravity, and $h=d / 2=r=$ distance or $1 \mathrm{D}$ length / 2).

For the minimum and maximum expansion of the Universe, the following points are considered:

- The variation of the capacity to perform work is always the same, limited by the perpetual existence of some empty space between masses (energy variation).

- Maximum expansion: two minimum masses (particles) are separated to maximum by $1 \mathrm{D}$ empty space, corresponding to $\left.\mathrm{E}_{0}=\mathrm{ETmax} E=\mathrm{Eg}_{\mathrm{E}}\right)$.

- Maximum contraction: the two minimum spaces are present, i.e., the universal structure is composed of three mass parts for the conservation of energy.

- $M_{0}-m_{0}=$ minimum mass (1D particle; where $M_{0}>m_{0}$, and $M_{0} \cong m_{0}$ ).

- $M_{0}+M_{0}+\left(M_{0}-m_{0}\right)=$ Universe total mass (no space in 1D space).

- The energy of the state of maximum contraction is the gravitational potential energy related to the masses existing $\left(M_{0}+m_{0}+\left(M_{0}-m_{0}\right)\right)$ and subtracted from the absence of 2 (Mo-mo), or the spaces present. 
- Energy available in the maximum contraction state (for expansion): $\left(M_{0}+M_{0}\right) \times c^{2}+\left(M_{0}-m_{0}\right) \times c^{2}-2\left(M_{0}-m_{0}\right) \times c^{2}=$ $\left(M_{0}+M_{0}\right) \times c^{2}-\left(M_{0}-m_{0}\right) \times c^{2}=\left(M_{0}+m_{0}\right) \times c^{2} ;$ formed by a maximum massive part, a minimum empty space, a minimum particle (mass), another minimum empty space, and another maximum massive part. (With the necessary presence of two internal empty spaces, corresponding to incomplete and maximum contraction for the energy conservation, $\mathrm{E}_{0}=\mathrm{EgC} \neq 0$ ).

- The energy of the state of maximum expansion is the gravitational potential energy related to the presence of empty space (1D), or the absence of masses: $\left(M_{0}+M_{0}+\left(M_{0}-m_{0}\right)\right)$, subtracted from the presence of two particles $(2(\mathrm{Mo}-\mathrm{mo}))$ that do not generate empty space or expansion.

- Energy available in the maximum expansion state (for contraction):

$\left(\left(M_{0}+M_{0}\right) \times c^{2}+\left(M_{0}-m_{0}\right) \times c^{2}-2\left(M_{0}-m_{0}\right) \times c^{2}\right)=$ $\left(\left(M_{0}+M_{0}\right) \times c^{2}-\left(M_{0}-m_{0}\right) \times c^{2}\right)=\left(M_{0}+m_{0}\right) \times c^{2}$

Two minimum massive particles separated to maximum by an empty space (1D) corresponding to incomplete and maximum expansion, for the energy conservation, $\mathrm{E}_{0}=\mathrm{EgE} \neq 0$ ).

In this way, the extreme universal states are represented as follows:

$$
\begin{aligned}
& \text { Energy available: } \mathrm{ETmaxC}=\mathrm{E}_{0}=\mathrm{EgC}=\left(\mathrm{M}_{0}+\mathrm{m}_{0}\right) \times \mathrm{c}^{2} \\
& \text { Energy available: } \mathrm{ETmax}=\mathrm{E}_{0}=\mathrm{EgE}=\left(\mathrm{M}_{0}+\mathrm{m}_{0}\right) \times \mathrm{c}^{2}
\end{aligned}
$$

$\mathrm{ETmaxC}=\mathrm{ETmax}$ (conservation of the energy available).

$$
\left(M_{0}+m_{0}\right) \times c^{2}=\left(M_{0}+m_{0}\right) \times c^{2} \quad(\text { Energy available })
$$

ETmaxE $=\left(M_{0}+m_{0}\right) \times c^{2}$, does not mean that the universe will have a rest mass quantity $\left(M_{0}+m_{0}\right)$ in its state of maximum expansion, but that it will have an energy corresponding to the universe at maximum contraction (the state with this rest mass present). 
In the linear states of maximum and minimum expansion, the gravitational potential energy is maximum and the same; in a state of maximum contraction, the amount of mass is maximum, but the distance to the center of mass is smaller (compared to the state of maximum expansion); in a state of maximum expansion, the amount of mass is minimum, but the distance from the particles to the center of mass is maximum.

The entropy (S) or the degree of freedom must be associated with the dimension; the degree is the same in possible extreme 1D space states $(\Delta S=0)$.

Therefore, expansions are followed by contractions of the same intensity (entire Universe)

Figs. 1-3 show that any contraction and expansion, although intense, cannot be total; demonstrating that all (entire) Universe is dynamic and is always transforming with the preservation of some energy variation.

(Example of a complementary model was elaborated only for an illustration).

Figure 1. Entire Universe.

\section{Entire Universe:}

(All existence $=$ Masses (energies) + empty space between masses) .

(Nothing) Entire Universe (maximum expansion, 1D space) (Nothing)

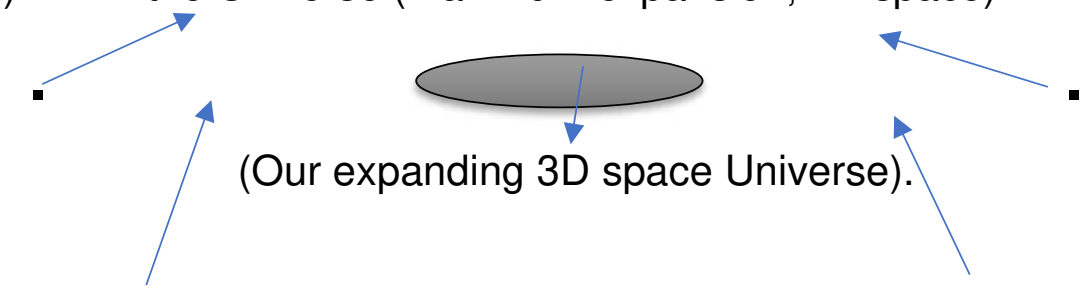

(The space that can exist by maximum expansion). 
Figure 2. Entire Universe (expansion).

\begin{tabular}{|c|c|c|c|}
\hline 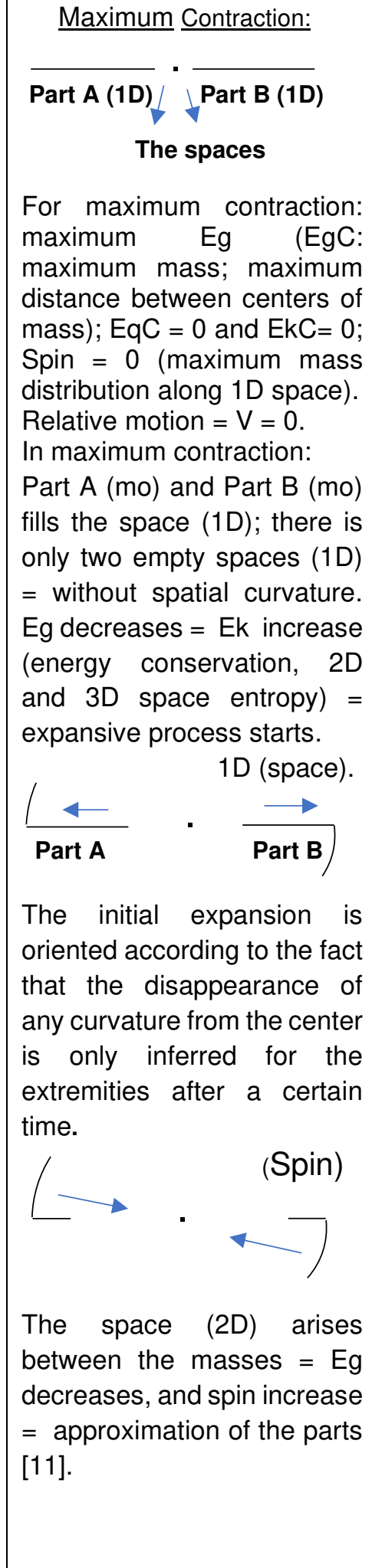 & $\begin{array}{l}\text { With the union of the } \\
\text { parts, the spin } \\
\text { increases, space arises } \\
\text { (for circular edge), } \\
\text { forming particles (with } \\
\text { 1D internal space). } \\
\text { The gravitational } \\
\text { attraction (edge) and the } \\
\text { spin are the cause for } \\
\text { the particles to arise } \\
\text { with different } \\
\text { accelerations, starting } \\
\text { an interaction. }\end{array}$ & $\begin{array}{l}\text { The interactions (two } \\
\text { dimensions), begin } \\
\text { to form particles with } \\
\text { the } 3 \mathrm{D} \text { internal } \\
\text { space, and thus } \\
\text { propagate into } 3 \mathrm{D} \\
\text { space. } \\
\text { With the formation of } \\
3 \mathrm{~d} \text { space, there is a } \\
\text { decrease in } 2 \mathrm{D} \\
\text { space. }\end{array}$ & $\begin{array}{l}\text { (Spin) } \\
\text { (Our Universe) } \\
\text { With the formation of 3D space } \\
\text { Universe, comes particles (3D) } \\
\text { at high temperatures or } \\
\text { radiation, space with little } \\
\text { interaction, and the inflation. } \\
\text { After inflation, the interactions } \\
\text { between particles increases } \\
\text { (temperature decrease). } \\
\text { The speed of expansion } \\
\text { decreases, causing more } \\
\text { particles (3D) to emerge. } \\
\text { With more intense interaction, } \\
\text { the amount of 1D and 2D } \\
\text { masses decreases, while those } \\
\text { of 3D increase, making the } \\
\text { expansion of the universe } \\
\text { accelerated with the increase of } \\
\text { its spin (by centrifugation). } \\
\text { A flat or curved [12] state of our } \\
\text { Universe (3D space) does not } \\
\text { predict the cyclic or non-cyclic } \\
\text { evolution of the entire Universe. } \\
\text { the extreme linear states of the } \\
\text { entire Universe (1D space) that } \\
\text { define the continuity of its } \\
\text { evolution. } \\
\text { Neutrino experiments (2020) } \\
\text { show the possible asymmetry in } \\
\text { the formation of matter and } \\
\text { antimatter [13]. Dark (matter and } \\
\text { energy) are 1D or } 2 D \text { particles } \\
\text { that have not yet interacted to } \\
\text { form 3D particles. }\end{array}$ \\
\hline
\end{tabular}


Figure 3. Entire Universe ( maximum expansion and contraction).

\begin{tabular}{|c|c|c|}
\hline 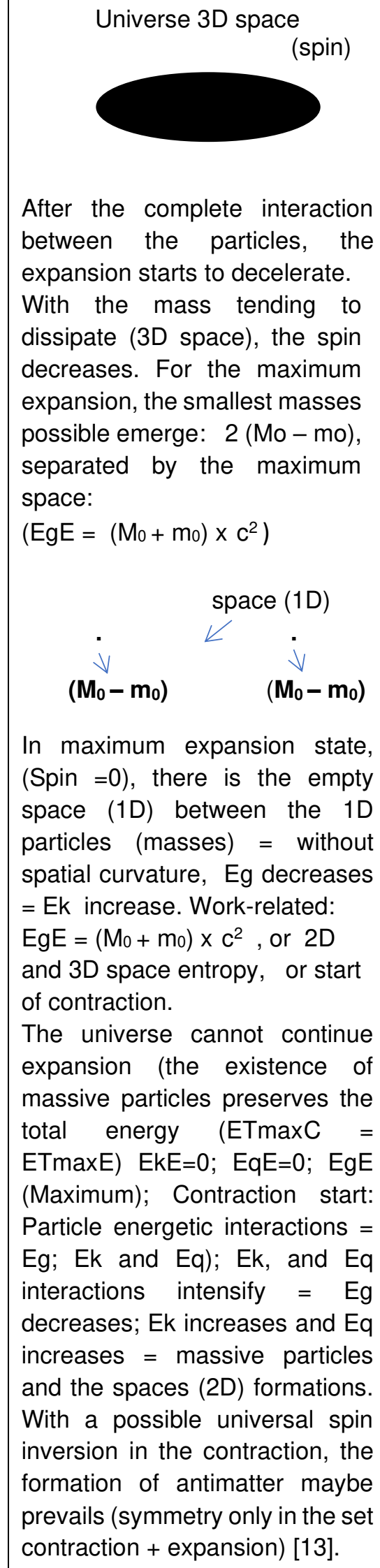 & $\begin{array}{l}\text { With Three Dimensional spaces } \\
\text { maximum compacted (The scarce } \\
\text { empty internal spaces) = } \\
\text { maximum spin (3D) }[15,16] \\
\text { Ek is not completely converted to } \\
\text { Eg (The contraction continue). Via } \\
\text { centrifugation and planarization, } \\
\text { will exist to a return to the second } \\
\text { dimension (2D). Maximum } \\
\text { massive contraction (2D) } \\
\text { dispersed in disc form. The } \\
\text { maximum contraction in 2D, will } \\
\text { consist of a fully compressed disk, } \\
\text { between two particles (symmetry), } \\
\text { so that there are spaces, for } \\
\text { potential energy (maximum mass } \\
\text { of disk) or energy conservation. }\end{array}$ & $\begin{array}{l}\text { In its maximum 2D contraction state, the } \\
\text { disk (with no internal spaces) loses its } \\
\text { internal gravitational effect. the contraction } \\
\text { continues (spin) to } 1 \mathrm{D} \text { space (Ek is not } \\
\text { completely converted to Eg). } \\
\text { When the disk loses particles by spin, the } \\
\text { only spaces that exists for motion is } \\
\text { between the edge of the disk and the } \\
\text { particles, that is, the surface (between the } \\
\text { disk and the particle) is cone shaped. } \\
\text { The spaces } \\
\text { Part A }\end{array}$ \\
\hline
\end{tabular}




\section{Conclusion}

It has been demonstrated that there can be maximum contraction to a single spatial dimension, but not so intense to be complete, tending only to a single point; similarly, maximum expansion can only occur without the existence of complete dissipation for the entire Universe; there is the perpetual existence of some empty space between masses (energy variation and conservation in the evolution of the entire universe).

The rest energy (Eo) in 1D (space) was considered, thus ruling out the use of certain relativistic and quantum calculations $(4 \mathrm{D}=3 \mathrm{D}($ space $)+1 \mathrm{D}$ (time) $)$ $[17,18]$.

The universe has finite energy (contraction and expansion are limited). Energy is limited one-dimensionally by the necessary presence of empty spaces at maximum contraction (1D space) and masses at maximum expansion (1D space).

For the beginning or end of any motion (expansion), the entire Universe must be at rest. Relative motion is zero only in the states of maximum contraction and expansion.

An equation has been formulated that respects the necessary preservation of total energy in the entire universe:

$$
\left(M_{0}+m_{0}\right) \times c^{2}=\left(M_{0}+m_{0}\right) \times c^{2}
$$


Our Universe (3D space), studied by relativity and quantum mechanics, does not conserve its energy total.

The smallest massive particles (formed by internal spaces 1D and 2D) and their associated energies are occupying and propagating in 3D space. while these particles do not bond to form 3D internal space particles, their presences are perceived by the gravitational, or energetic effect of their motions. When they group together to form 3D particles, with the increased energy effect, the acceleration of the expansion is enhanced, by the faster spin of our Universe.

The current motion of the Universe already represents an energetic and spatial variation.

Space analysis (1D and 2D) was used to build a complementary universal model in which the details are supported by some concepts already well established in physics, and others, necessary for the interpretation of observational data.

The model presented can demonstrate why the universe starts its spin with the beginning of the expansion [11].

Every energy signal is absorbed in the maximum contraction state of each cycle; thus, Olbers' paradox [19] can be disregarded.

This approach respects the Ockham razor perspective and the principle of parsimony. The best explanation should assume the fewest number of premises [20]. 


\section{References}

[1] Universe. Webster`s New World College Dictionary. Wiley Publishing, Inc.

[S.I.: s.n.]. 2010

[2] Souza Y.L. Cosmologia (Cosmology). Infoescola (2018)

[3] Stephen H., Bizi F., Ellis G.F.R.: The large-scale structure of space-time.

Cambridge, Cambridge University Press (1973)

[4] Petit J.P., d'Agostini G.: Cancellation of the central singularity of the

Schwarzschild solution with natural mass inversion process Mod. Phys Lett A 30, 9 (2015)

[5] Neves, J.C.S.: Bouncing cosmology inspired by regular black holes.

Gen.Rel. Grav, 49,124 (2017)

[6] Baptista J.P.: Os princípios fundamentais ao longo da história da física.

Rev. Bras. Ensino Fís. 28, 541-553 (2006)

[7] Mureika J., Stojkovic D.: Detecting Vanishing Dimensions via Primordial Gravitational Wave Astronomy. Physical Review Letters, Vol.: 106, 101101, March (2011)

[8] Anchordoqui L., Dai De Chang, Fairbairn M., Landsberg G., Stojkovic D.: Vanishing Dimensions and Planar Events LHC.

arXiv: 1003,5914. (2011)

[9] Jain C.M.: Textbook of engineering physics. S.I.: Phi learning (2009)

[10] Vieira S., Barros A., Araújo I., Oliveira J.C.T.: A comparison among deductions of the equation $E=m c^{2}$. Rev. Bras. Ensino Fís. 26, 93-98 (2004) [11] Wang, P. Libeskind, N.T., Tempel, E.et al. Possible observational evidence for cosmic filament spin. Nat Astron (2021).

[12] Valentino, E.D., Melchiorri A., Silk J.: Planck evidence for a closed Universe and a possible crisis for cosmology. Nature Astronomy 4, 196203 (2020)

[13] The TK Collaboration, Constraint on the matter-antimatter symmetryviolating phase in neutrino oscillations. Nature. 580, 339-344 (2020)

[14] Truesdell C.A.: The tragicomical history of thermodynamics, Springer, New York, (1980)

[15] Walker, J. Fundamentos de Física. 10th Ed. Rio de Janeiro, LTC (2016) 
[16] Ramalho N.T.: Os Fundamentos da Física - Moderna Plus. Vol. 01. Moderna. 11를 Ed. SP (2016)

[17] O'Connor J.J., Robertson E.F.: General relativity. Mathematical Physics Index. 1996. Esc. Mat. Estatística Univ. St. Andrews (1996) [18] Roberto B., Netto L.F.: What is the special theory of relativity. Science meeting. (O que é a Teoria da Relatividade Especial. Feira de Ciências (2009) [19] Souza R.E.: Introduction to cosmology (Introdução à Cosmologia). São Paulo, Edusp (2004)

[20] Ariew R.: Ockham's razor: a historical and philosophical analysis of Ockham's principle of parsimony. Champaign-Urbana, University of Illinois (1976) 